EPJ Web of Conferences 23, 00019 (2012)

DOI: $10.1051 /$ epjconf/20122300019

(C) Owned by the authors, published by EDP Sciences, 2012

\title{
Strongly correlated electron behavior in carbon nanotubes
}

\author{
Marc Bockrath ${ }^{1, a}$ Vikram V. Deshpande ${ }^{2}$, Bhupesh Chandra ${ }^{3}$, Robert Caldwell ${ }^{2}$, Dmitry S. Novikov ${ }^{4}$, and James Hone ${ }^{2}$ \\ 1 University of California, Riverside, CA \\ 2 Columbia University, New York, NY \\ 3 IBM TJ Watson Research Center, Yorktown Heights, NY \\ 4 New York University School of Medicine, New York, NY
}

\begin{abstract}
One dimensional systems offer a fascinating platform for investigating and understanding the collective and many-body behavior of interacting electron systems. We report low-temperature transport experiments on carbon nanotubes, which are archetypal one-dimensional systems that have either semiconducting or metallic band structure depending on their radius and chirality. Semiconducting nanotubes at low densities exhibit Wigner crystal behavior, while nominally metallic nanotubes are observed to have an energy gap at half filling, consistent with theories of a Mott insulating state in nanotubes. Our results demonstrate nanotubes' promise for studying a variety of tunable correlated electron phenomena in one dimension.
\end{abstract}

\section{Introduction}

Many properties of three dimensional metals can be understood on the basis of Fermi liquid theory in which the low-energy excitations behave as weakly interacting quasiparticles with similar properties to free electrons. As electrons are confined to fewer dimensions, the effects of interactions become greater. In one-dimensional (1D) systems, Fermi liquid theory breaks down, the ground state of the system is instead described by a Luttinger liquid, in which the low energy excitations are not quasiparticles but instead bosonic waves of charge and spin[1-3]. One of the hallmarks of a Luttinger liquid is that tunneling into a Luttinger liquid is expected to be suppressed at low energy, leading to a zero-bias anomaly in the differential conductance[4]. Another is the phenomena of spin-charge separation, in which the spin and charge modes propagate at different velocities[1]. The ratio of the spin wave velocity to the charge wave velocity can be used to parameterize the strength of the interactions and is typically denoted by $g$, where $g<1$ for repulsive interactions. The zerobias anomaly has now been observed in a number of systems, including carbon nanotubes, while spin-charge separation has been observed in cleaved edge overgrowth systems[5]. A qualitatively different regime of the Luttinger liquid emerges when the charges are at sufficiently low density that the exchange energy becomes less than the Fermi energy. In this case, the electrons are expected to periodically order to form a solid-like state of electron matter which is a $1 \mathrm{D}$ Wigner crystal[6-8]. One can view the Wigner crystal state as arising from a competition between the kinetic energy of the electrons which is given by $K \sim$ $E_{F} \sim \hbar n^{2} / m$, where $\hbar$ is Plank's constant divided by $2 \pi$, $m$ is the electron effective mass, and $n$ is the electron density, and their potential energy which is given by $P \sim e^{2} / d$, where $d$ is the mean electron separation, and $e$ is the electric charge. When the kinetic energy is small compared to

\footnotetext{
a e-mail: marc.bockrath@ucr.edu
}

the potential energy the electrons are unable to exchange positions classically, and therefore periodically order to minimize their Coulomb energy. The ratio $K / P$ can be written as $n a_{B}$, where $a_{B}$ is the Bohr radius, which indicates that the Wigner crystal state is favored at low densities. While the electrons are forbidden to classically exchange positions, they are allowed to quantum mechanically exchange positions by tunneling, which gives rise to antiferromagnetic exchange interactions between the electrons [68]. A Wigner crystal thus behaves similarly to a spin chain[6$8]$.

Adding a lattice to a $1 \mathrm{D}$ system can produce backscattering via umklapp processes. When the lattice is commensurate with the mean electron spacing this can favor the development of an energy gap at the Fermi level. An example of this occurs when there is one electron per atomic site in a $1 \mathrm{D}$ chain. One can model the interactions by assuming an energy cost $U$ to add more than one electron per site, and also assuming that the electron can move through the lattice with a nearest-neighbor hopping parameter $t$. In the limit $U / t>>1$, creating an electron hole pair costs an energy $U$ and thus the system is an insulator. It can be shown that unlike the 3D case where interaction must be sufficiently strong in order to open a gap[9], in 1D an energy gap exists for any $U / t>0[1]$.

\section{Carbon nanotubes as 1D interacting electron systems}

Carbon nanotubes have emerged as nearly ideal systems in which to study the physics of electrons confined to one dimension[10]. Carbon nanotubes are derived from graphene, a honeycomb lattice of carbon atoms (Fig 1a, left). Their low energy band structure consists of cones with gapless points at the Fermi energy $E_{F}$ (Fig 1a, right). Carbon nanotubes can be conceived of as having been rolled from the graphene sheet to produce a tube; the electronic properties depend on precisely how the nanotube is rolled, that is on 
(a)

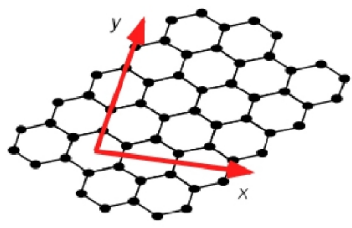

armchair

(b)

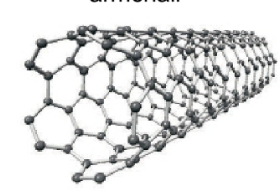

(c)

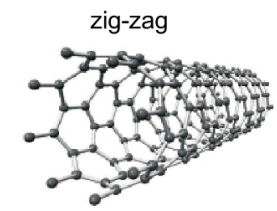

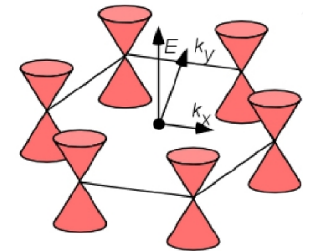
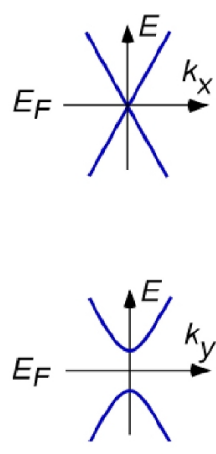

Fig. 1. Band structure of carbon nanotubes. (a) Left: the lattice of graphene, right: the band structure of graphene near the Fermi level. (b) Left: the structure of an armchair nanotube, right: its corresponding band structure. (c) Left: the structure of a zig-zag nanotube, right: its corresponding band structure.

its radius and orientation relative to the honeycomb lattice. The type of nanotube is specified using two integers $(N, M)$ that indicate a vector connecting two hexagons that are identified for each different possible wrapping. Since the electron wavefunction must be single valued and return to the same value when completing a circuit around the nanotube, the allowed momenta are quantized within the twodimensional graphene Brillouin zone to form 1D bands. In the simplest picture, the nanotube is metallic if the allowed momenta include the gapless points and semiconducting otherwise. A $(5,5)$ armchair nanotube is shown in Fig. 1b, which is metallic, and an $(8,0)$ zig-zag structure in Fig 1c, which is semiconducting. Single-walled nanotubes are typically $\sim 1-3 \mathrm{~nm}$ in diameter. Because of their small diameter, the energy spacing between transverse modes is $\sim 1 \mathrm{eV}$, and therefore nanotubes are effectively $1 \mathrm{D}$ systems. Because of the two inequivalent Dirac points, the states in nanotubes have both a valley and spin degeneracy, yielding a four-fold degeneracy[10].

One powerful technique for investigating the properties of carbon nanotubes is Coulomb blockade spectroscopy. In Coulomb blockade the nanotube forms an island for charge, separated from attached electrodes by tunnel barriers. Because of Coulomb repulsion, there is a charging energy $E_{C}$ to add an extra electron on the dot. Thus the electrons can be added one at a time by adjusting the voltage on a nearby gate electrode. Each time an electron is added, a peak is produced in the conductance. The spacing in gate voltage between these peaks reflects the energy cost to add each electron, and measurement of the energy for the $n$-electron ground states yields much information about how the electrons behave within the nanotubes. The energies of the electron states can be altered by applying a magnetic field along the axis. The electrons' sense of orbit around the nanotube determines their orbital magnetic moment which can couple to the magnetic field. The sense of orbit, whether clockwise or counterclockwise

(a)

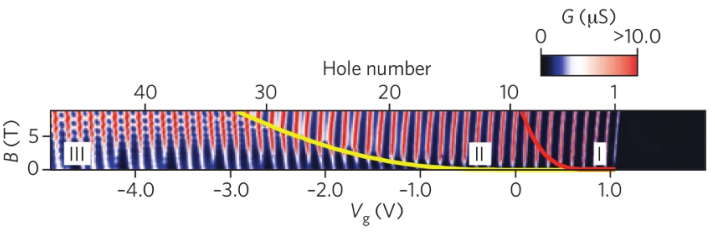

(b)

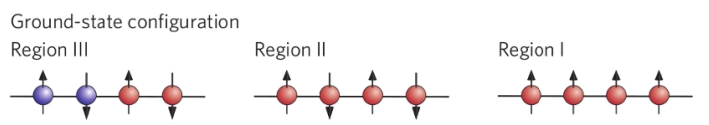

Fig. 2. Wigner crystal physics in a carbon nanotube. (a) Color plot of conductance versus gate voltage and axial magnetic field. Bright features are Coulomb peaks. The evolution of the peaks fall into three distinct regimes, labeled I, II and III, as discussed in the text. (b) Electron configuration for the three different regimes in the Wigner crystal regime.

can be labeled by an isospin quantum number; the magnetic field splits these degenerate isospin states[11] by an amount proportional to the nanotube radius. In addition, the spin states are split by the Zeeman effect[12,13], which typically yields a splitting 5-10 times smaller than the isospin splitting[11].

Figure 2a shows a color-scale plot of conductance versus gate voltage and magnetic taken from a $0.5 \mu \mathrm{m}$ long nanotube which exhibits Coulomb blockade behavior. The Coulomb peaks shift position as the magnetic field is varied. Three regions are evident. In region I, the Coulomb peaks follow parallel evolution with a parallel slope. In region II, the peaks exhibit an alternating slope, and in region III, the peaks follow a more complex zig-zag evolution. The parallel slope of the Coulomb peaks' motion with magnetic field in region I shows that the charges are entering the nanotube with the same spin and isospin, This is difficult to understand in a conventional shell filling picture, which would predict few or no spin polarized electrons in region I[14]. In the Wigner crystal picture, the ground state is a competition between the magnetic energy, which tends to align spins and the exchange which favors antiparallel alignment. The exchange energy is strongly dependent on the charge density, becoming larger as the density is increased. Thus the three regions observed in Fig. 2a can be understood on the basis of the diagrams shown in Fig. 2b. Region I corresponds to a fully polarized spin and isospin chain, region II corresponds to a isospin polarized, antiferromagnetically ordered chain, and region III corresponds to a minimally polarized chain. The yellow line in Fig. 2a shows a single-parameter fit to theory, using the effective Bohr radius $a_{B}^{*}$ as a fit parameter. The resulting best fit $a_{B}^{*} \sim 10 \mathrm{~nm}$, which lies within the theoretically predicted range of $a_{B}^{*} \sim 5-20 \mathrm{~nm}$ for this nanotube. The red line is a plot of the boundary between regions I and II made using the same parameter value, showing satisfactory agreement between experiment and theory[14]. The quantitative agreement between theory and experiment provides strong evidence that electrons in semiconducting carbon nanotubes are in a $1 \mathrm{D}$ Wigner crystal state at low densities.

Another interesting situation occurs with carbon nanotubes that are expected to be metallic by their structure. It has been recognized that many such nanotubes can have a small gap opened by their curvature[10]. The gap scales 
(a)

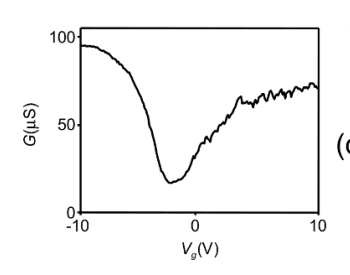

(b)

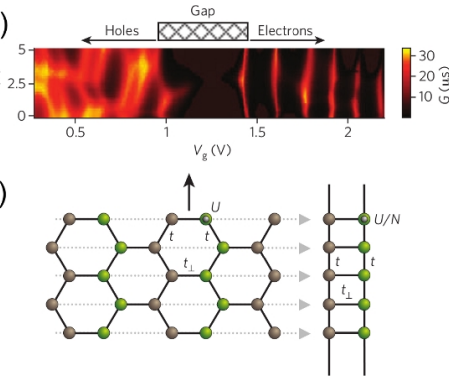

Fig. 3. Mott insulating behavior in carbon nanotubes. (a) Conductance versus gate voltage for an armchair nanotube, showing a dip near zero gate voltage. (b) Color plot of conductance versus gate voltage and axial magnetic field. As the magnetic field increases, the gap closes, reaches a non-zero minimum, then opens again. (c) Mapping of an $(N, N)$ armchair nanotube onto an effective two-leg ladder. Here $t$ and $t_{\perp}$ are hopping parameters, while $U$ is the on-site interaction.

as $1 / r^{2}$ where $r$ is the nanotube radius, and depends on the chiral angle. For the armchair nanotube structure, the symmetry prohibits the opening of a curvature induced gap. Nevertheless, experiments on armchair nanotubes show a conductance dip (Fig. 3a). It has been demonstrated that the conductance minimum shows an activated temperature dependence, signifying the presence of a gap[15]. Other gap opening mechanisms involve breaking the symmetry of the nanotube in some way, for example by a longitudinal strain or a twist. These mechanisms operate by shifting the Dirac point of the graphene sheet away from the lines of allowed momenta[10]. The lines of allowed momenta in the Brillouin zone can be shifted by an axial magnetic field via the Aharonov-Bohm effect $[11,16]$. Thus we expect if the above gap opening mechanisms were responsible for the observed gap, the gap should be able to be closed by the application of a sufficiently large axial magnetic field. Instead, as shown in Fig. 3b, the gap begins to close, reaches a minimum, non-zero value, and then begins to open again. This behavior is inconsistent with the above models for gap opening in nanotubes. Theory predicts, however, that nominally metallic nanotubes can have a gap opened due to the formation of a 1D Mott insulator[17-22]. The structure of the nanotube can be mapped onto a two-leg ladder[18], as shown in Fig. 3c. In this mapping the bare $U$ interaction is scaled by a factor of $1 / N$ for an $(N, N)$ nanotube. Thus the bare interaction scales as $1 / r$. Theory that takes into account long ranged interactions shows that the energy gap should go as $\Delta \sim 1 / r^{\beta}$ for all metallic nanotubes regardless of structure, where $\beta$ ranges from $1 /(1-g)$ to 2 , depending on the detailed assumptions of the theory[1722]. Experimental results show that $\beta \sim 1.3[15]$, within the range of theoretically predicted exponents. In addition, experiments show the presence of low energy excitations within the gap[15], which are also in accordance with theory $[19,21]$. This provides additional evidence that the gap observed in metallic nanotubes results from Mott insulator physics.

In conclusion, carbon nanotubes are a fascinating system in which to study many body physics in one dimension. Many properties of nanotubes require going beyond non-interacting single particle models in order to understand. For example, one dimensional Wigner crystal physics and 1D Mott insulating states have been observed. De- spite this progress, many theoretical predictions remain to be tested, for example, concerning spin incoherent[6] and non-linear Luttinger liquids[23]. Carbon nanotubes will no doubt continue to provide an exciting system that yields experimental insight into the nature on interacting electrons in $1 \mathrm{D}$.

\section{References}

1. T. Giamarchi, Quantum Physics in One Dimension (Oxford University Press, 2004).

2. J.M. Luttinger, J. Math. Phys. 4, 1154 (1963).

3. S. Tomonaga, Progr. Theoret. Phys. 5, 544 (1950).

4. C.L. Kane, M.P.A. Fisher, Phys. Rev. Lett. 68, 1220 (1992).

5. V.V. Deshpande, M. Bockrath, L.I. Glazman, A. Yacoby, Nature 464, 209 (2010).

6. G.A. Fiete, Rev. Mod. Phys. 79, 801 (2007).

7. K.A. Matveev, Phys. Rev. Lett 92, 106801 (2004).

8. D.V. Averin, Y.V. Nazarov, Phys. Rev. B 47, 9944 (1993).

9. N.F. Mott, Rev. Mod. Phys. 40, 677 (1968).

10. J. Charlier, X. Blase, S. Roche, Rev. Mod. Phys. 79, 677 (2007).

11. E.D. Minot, Y. Yaish, V. Sazonova, P.L. McEuen, Nature 428, 536 (2004).

12. D.H. Cobden, M. Bockrath, P.L. McEuen, A.G. Rinzler, R.E. Smalley, Phys. Rev. Lett. 81, 681 (1998).

13. S.J. Tans, M.H. Devoret, R.J.A. Groeneveld, C. Dekker, Nature 394, 761 (1998).

14. V.V. Deshpande, M. Bockrath, Nat. Phys. 4, 314 (2008).

15. V.V. Deshpande, B. Chandra, R. Caldwell, D.S. Novikov, J. Hone, M. Bockrath, Science 323, 106 (2009).

16. U.C. Coskun, T.C. Wei, S. Vishveshwara, P.M. Goldbart, A. Bezryadin, Science 304, 1132 (2004).

17. C. Kane, L. Balents, M.P.A. Fisher, Phys. Rev. Lett. 79, 5086 (1997).

18. L. Balents, M.P.A. Fisher, Phys. Rev. B 55, R11973 (1997).

19. A.A. Odintsov, H. Yoshioka, Phys. Rev. B 59, 10457 (1999).

20. Y.A. Krotov, D.H. Lee, S.G. Louie, Phys. Rev. Lett. 78, 4245 (1997).

21. A.A. Nersesyan, A.M. Tsvelik, Phys. Rev. B 68, 235419 (2003).

22. W. Chen, A.V. Andreev, A.M. Tsvelik, O. Dror, Phys. Rev. Lett. 101, 246802 (2008).

23. M. Pustilnik, M. Khodas, A. Kamenev, L.I. Glazman, Phys. Rev. Lett. 96, 196405 (2006). 